\title{
Epigenome-Wide DNA Methylation Analysis of Monozygotic Twins Discordant for Diurnal Preference
}

\author{
Chloe C. Y. Wong, ${ }^{1}$ Michael J. Parsons, ${ }^{2}$ Kathryn J. Lester, ${ }^{1,3}$ Joe Burrage, ${ }^{4}$ Thalia C. Eley, ${ }^{1}$ \\ Jonathan Mill, ${ }^{1,4}$ Emma L. Dempster, ${ }^{4 *}$ and Alice M. Gregory ${ }^{5 *}$ \\ ${ }^{1}$ Institute of Psychiatry, Psychology \& Neuroscience, King's College London, MRC Social, Genetic \& Developmental \\ Psychiatry Centre, London, UK \\ ${ }^{2}$ Mammalian Genetics Unit, MRC Harwell, Harwell Science and Innovation Campus, Oxfordshire, UK \\ ${ }^{3}$ School of Psychology, University of Sussex, Brighton, UK \\ ${ }^{4}$ University of Exeter Medical School, University of Exeter, Exeter, UK \\ ${ }^{5}$ Department of Psychology, Goldsmiths, University of London, London, UK
}

\begin{abstract}
Diurnal preference is an individual's preference for daily activities and sleep timing and is strongly correlated with the underlying circadian clock and the sleep-wake cycle validating its use as an indirect circadian measure in humans. Recent research has implicated DNA methylation as a mechanism involved in the regulation of the circadian clock system in humans and other mammals. In order to evaluate the extent of epigenetic differences associated with diurnal preference, we examined genome-wide patterns of DNA methylation in DNA from monozygotic (MZ) twin-pairs discordant for diurnal preference. MZ twins were selected from a longitudinal twin study designed to investigate the interplay of genetic and environmental factors in the development of emotional and behavioral difficulties. Fifteen pairs of MZ twins were identified in which one member scored considerably higher on the Horne-Ostberg Morningness-Eveningness Questionnaire (MEQ) than the other. Genome-wide DNA methylation patterns were assessed in twins' buccal cell DNA using the Illumina Infinium HumanMethylation450 BeadChips. Quality control and data pre-processing was undertaken using the wateRmelon package. Differentially methylated probes (DMPs) were identified using an analysis strategy taking into account both the significance and the magnitude of DNA methylation differences. Our data indicate that DNA methylation differences are detectable in MZ twins discordant for diurnal preference. Moreover, downstream gene ontology (GO) enrichment analysis on the top-ranked diurnal preference associated DMPs revealed significant enrichment of pathways that have been previously associated with circadian rhythm regulation, including cell adhesion processes and calcium ion binding.
\end{abstract}

Keywords: epigenetics, DNA methylation, diurnal preference, circadian rhythm, monozygotic twins

Circadian rhythms are endogenous biological processes that adhere to a cycle of approximately 24 hours and regulate a wide variety of key physiological and metabolic processes in all living organisms, including the sleep-wake cycle (Dijk \& Lockley, 2002). In mammals, circadian rhythms are driven by a central pacemaker in the hypothalamus, itself driven by a complex molecular clock (Zhang \& Kay, 2010). As measuring the intrinsic properties of the central pacemaker in humans is difficult and time-consuming, indirect measures are more commonly used, such as diurnal preference. Diurnal preference is an individual's preference for timing of daily activities and sleep and can be measured via a self-reported questionnaire that yields a numerical score on a scale ranging between extreme morning preference and extreme evening preference (Horne \& Ostberg, 1976). The relationship between diurnal preference and circadian period is highly correlated, and that diurnal preference is a well-validated indirect measure of circadian function (Duffy et al., 2001; Mongrain et al., 2004; Zhang \& Kay, 2010).

RECEIVED 11 September 2015; ACCEPTED 1 October 2015. First published online 20 November 2015.

ADDRESS FOR CORRESPONDENCE: Emma L. Dempster, University of Exeter Medical School, University of Exeter, Exeter, UK. E-mail: e.l.dempster@exeter.ac.uk

* These authors contributed equally 
Diurnal preference varies between individuals (Kerkhof, 1985), with quantitative genetic studies determining that this variation is influenced by both genetic and environmental factors; genetic variation accounts for around 50\% of variance in diurnal preference, with the remaining variance accounted for by non-shared environmental influence (Barclay et al., 2010; Koskenvuo et al., 2007). Specifically, a recent study using a cohort of $420 \mathrm{MZ}$ twins, 773 dizygotic twins, and 329 siblings from a population-based twin registry across the United Kingdom (including samples used for this study) reported that dominance genetic influence accounted for $52 \%$ and non-shared environment $48 \%$ of variance in diurnal preference (Barclay et al., 2010). A number of genes and related pathways have been significantly associated with mammalian sleep regulation and diurnal preference, including those encoding for ion channels, circadian rhythms, cellular growth signals, neurotransmitters and their receptors, as well as cytokines, and stress responses, to name a few (Archer et al., 2010; Katzenberg et al., 1998; Palagini et al., 2014; Toh et al., 2001). In addition to genetic and environmental influences, diurnal preference varies by sex, where eveningness is significantly associated with males (von Schantz et al., 2015). Eveningness has also been associated with a range of behavioral and emotional problems (Gau et al., 2004, 2007) as well as adolescence and adult depression (Gau et al., 2007; Randler, 2011; Wittmann et al., 2010).

Epigenetic mechanisms such as DNA methylation are dynamic biological processes that have the potential to mediate both genetic and environmental influences on circadian processes (Azzi et al., 2014; Feng et al., 2011; Qureshi \& Mehler, 2014). Emerging evidence implicates a role for epigenetic mechanisms in transcriptional and posttranscription regulation within the circadian system (Azzi et al., 2014; Belden et al., 2011; Feng et al., 2011). One of the most powerful designs in epigenetic epidemiology is the use of phenotype-discordant MZ twins because identical twins are matched for genotype, age, sex, maternal influences, population cohort effects, and exposure to many shared environmental factors (Bell \& Spector, 2011; Heijmans \& Mill, 2012).

In this study, we examined genome-wide patterns of DNA methylation in buccal cell derived DNA from MZ twin-pairs discordant for diurnal preference in order to identify epigenetic variation associated with diurnal preference. This is the first study, to our knowledge, to investigate the associations between differential DNA methylation and diurnal preference in human subjects.

\section{Subjects and Method Study Cohort}

Participants were selected from the Genesis 12-19 (G1219) study, a longitudinal twin and sibling study designed to investigate the development of emotional and behavioral difficulties, focusing on the interplay between genes and environment (McAdams et al., 2013). The twins were recruited via the United Kingdom Office of National Statistics, and five waves of data collection have been collected to date. At wave 1, informed consent was obtained from parents/guardians of all adolescents $<16$ years and from adolescents themselves when 16 years or older. The Research Ethics Committees of the Institute of Psychiatry, King's College London, South London and Maudsley NHS Trust and Goldsmiths College, University of London, provided ethical approval for the different waves of data collection. This study focuses exclusively on the MEQ (as a measure of diurnal preference; Horne \& Ostberg, 1976) data collected at Wave 4 (collected in 2007). The MEQ is a 19-item selfreport questionnaire that assesses individual preference in the timing of daytime activities, sleeping habits, hours of peak performance, and times of 'feeling best', and maximum alertness. Individual items are rated on a 4- or 5-point scale, with the responses summed to give a total score on the morningness-eveningness dimension ranging from 16 to 86. Lower scores indicate greater 'eveningness'. Within G1219, the MEQ has been shown to have good reliability and validity (Barclay et al., 2010). MZ twin pairs were considered for inclusion if both twins had provided a DNA sample (obtained from buccal cells) and MEQ data ( $n=149$ $M Z$ pairs). The mean MEQ difference score for this whole sample from the G1219 cohort was $6.03(S D=4.75)$. For this study, we designed a within-twin difference score of 10 as representing sufficient discordancy to be of interest, and identified a total of $15 \mathrm{MZ}$ twin pairs (mean age at DNA collection $=17.96$ years, $S D=2.64$; mean age at MEQ questionnaire collection $=19.40$ years, $S D=1.07$; eight female pairs, seven male pairs, 93\% White European). Specifically, the mean difference score for this sample was 13.43 ( $S D=$ 2.04) and the mean MEQ score for the twin-pair member with greater eveningness was $44.05(S D=6.92)$, whereas the co-twin group mean MEQ score was $57.47(S D=6.10)$. It is worth noting that none of the discordant twin-pairs identified for this study reached the criteria for depression discordance used in our previous study of Dempster et al. (2014). The mean difference in the depression score (SMFQ) for the 15 pairs of diurnal preference discordant twins was $5.8(S D<3.5)$. In addition, none of the discordant diurnal preference twin-pairs were discordant for anxiety symptoms using the same cut-off (i.e., a discrepancy of $S D<3.5)$. Furthermore, there were no discrepant cases even when this criteria was relaxed $(<2.5$ times the $S D$ of the within twin difference across the whole G1219 samples measured using the adapted Revised Symptoms of Anxiety Scale as described in (Gregory et al., 2011)). The mean difference in the anxiety score for the 15 pairs of diurnal preference discordant twins was $9.4(S D=6.39)$. DNA was obtained from buccal cells and isolated using a standard protocol (Freeman et al., 2003). 


\section{DNA Methylomic Profiling}

Genomic DNA (500 ng) from each sample was treated with sodium bisulfite in duplicate using the EZ-96 DNA methylation kit (Zymo Research Corporation, Irvine, California). Genome-wide DNA methylation was quantified using the Illumina Infinium ${ }^{\circledR}$ HumanMethylation450 BeadChip (Illumina $450 \mathrm{~K}$ array) (Illumina, San Diego, CA, USA) and scanned on the HiScan System (Illumina, San Diego, CA, USA) as previously described (Pidsley et al., 2013). Illumina GenomeStudio software (Illumina, San Diego, CA, USA) was used to extract signal intensities for each probe, generating a final report that was imported into the $\mathrm{R}$ statistical environment 3.0.2 (www.r-project.org) using the methylumi package (Davis et al., 2015). Data quality control and pre-processing were performed using 'dasen' from the $w a$ teRmelon package as described previously (Pidsley et al., 2013). Specifically, 'dasen' involves quantile normalization of the data (which adjusts for background differences between Type I and Type II probes) and then between-array normalization to these probes separately with no dye bias correction. Stringent filtering of the pre-normalized Illumina $450 \mathrm{~K}$ array data was performed, where probes with $>1 \%$ of samples having a detection $p$ value $>.05$ were removed and only twin-pairs where both samples had $<5 \%$ of probes with a detection $p$ value $>.05$ were included in the analysis. Given the genetically sensitive design of this study where $\mathrm{MZ}$ twin-pairs are matched for genetic variation and sex, probes containing single-nucleotide polymorphisms within probe sequences were retained for downstream analyses but potential non-specific probes as identified by Chen et al. (2012) and Price et al. (2013) have been flagged in all result tables. The final analyses included 454,229 probes and all samples passed our stringent quality control filter. Polymorphic single nucleotide polymorphism control probes $(n=65)$ located on the array were used to confirm that all twin-pairs were $\mathrm{MZ}$.

\section{Data Analyses}

All statistical analyses were performed using the R statistical package (version 3.1.1). With the aim of identifying real, biologically relevant within-twin, and between-group DNA methylation differences, we used an analytic approach that incorporates both the significance (i.e., paired $t$-test statistic) and the magnitude (i.e., absolute $\Delta \beta$ ) of any observed differences to produce a ranked list of DMPs (Dempster et al., 2011; Wong et al., 2014). Specifically, probes were ranked separately by paired $t$-test, $p$ values, and $\Delta \beta$, and the ranks were summed. Additional permutated $p$ values were calculated by 10,000 permutations (i.e., random shuffling of phenotype labels) per probe. Differentially methylated region (DMRs) analysis was performed across 500bp regions using comb-p (Pedersen et al., 2012), a Python module that groups spatially correlated CpGs in user-defined sliding windows.

\section{Gene Ontology Term Enrichment Analysis}

Exploratory downstream GO term enrichment analysis was performed on the genes associated with the top 500 ranked DMPs using the R package GOseqv1.18.1 (downloaded from Bioconductor; Young et al., 2010). GOseq can be used to correct for the number of Illumina $450 \mathrm{k}$ probes in each gene during GO term enrichment analysis. The number of probes per gene was calculated in our final dataset to create a probability weighting function, which was then used in the GO term enrichment analysis.

\section{DMP Validation Using Bisulfite Pyrosequencing}

Although the Illumina $450 \mathrm{~K}$ array has been well validated for detecting differences in DNA methylation (Sandoval et al., 2011), we further tested a specific region nominated from the genome-wide microarray analysis using bisulfite pyrosequencing. Independent verification analysis was performed on the probe cg10960055, which is located in a cluster of tRNA genes located at $6 \mathrm{p} 22.1$. This probe demonstrated an average decrease in DNA methylation of $8 \%$ in the twin-pairs with high eveningness preference as compared to their low eveningness preference co-twins. Briefly, 500 ng DNA from each individual was independently treated with sodium bisulfite in duplicate using the EZ 96-DNA methylation kit as described earlier. Bisulfite-polymerase chain reaction amplification was performed in duplicate, and quantitative DNA methylation analysis was conducted using the PyroMark Q24 pyrosequencer (Qiagen, Valencia, California). The primers and assay condition are given in Table S1.

\section{Results}

Site-Specific Differential DNA Methylation in MZ Twins Discordant for Diurnal Preference

We assessed genome-wide patterns of DNA methylation in MZ twins discordant for diurnal preference using the $450 \mathrm{~K}$ arrays and an analytical approach designed to identify the largest and most significant differences in DNA methylation at individual $\mathrm{CpG}$ sites. As expected, we found no evidence of significant difference in overall mean genomewide DNA methylation between discordant twins for diurnal preference $(p=.74)$, indicating that diurnal preference is not associated with any global changes in the methylome. In contrast, we identified multiple CpG sites across the genome exhibiting nominally significant diurnal preference-associated differential DNA methylation. Table 1 lists the 10 top-ranked DMPs associated with diurnal preference, where consistent within-twin differences in DNA methylation were observed across the majority of discordant MZ twin-pairs investigated (Figure 1). The top ranked DMP, cg07409153 $(\Delta \beta 0.06, p=4.27 \mathrm{E}-$ 04 ) is located on chromosome $2 \mathrm{p} 12$, upstream of GCFC2 (gene encoding GC-Rich Sequence DNA-Binding Factor 2) 
TABLE 1

The Ten Top-Ranked DMPs in MZ Twins Discordant for Diurnal Preference

\begin{tabular}{|c|c|c|c|c|c|c|c|}
\hline Rank & $\begin{array}{l}\text { Probe } \\
\text { ID }\end{array}$ & $p$ value & $\begin{array}{l}\text { Empirical } p \text { value } \\
\text { (10000 permutation) }\end{array}$ & $\begin{array}{l}\text { Mean } \\
\Delta \beta\end{array}$ & $\begin{array}{l}\text { Genomic } \\
\text { coordinate (hg19) }\end{array}$ & $\begin{array}{l}\text { Illumina gene } \\
\text { annotation }\end{array}$ & $\begin{array}{l}\text { Gene annotation from GREAT } \\
\text { (Distance from TSS) }\end{array}$ \\
\hline 1 & cg07409153 & 4.27E-04 & $9.00 \mathrm{E}-04$ & 0.062 & chr2:76839078 & & GCFC2 (-900968), LRRTM4 (+910423) \\
\hline 2 & cg23881566 & 7.29E-04 & $1.80 \mathrm{E}-03$ & -0.063 & chr12:119457146 & SRRM4 & HSPB8 (-159448), SRRM4 (+37847) \\
\hline 3 & cg06142043 & $1.01 \mathrm{E}-03$ & 1.50E-03 & 0.064 & chr6:8064743 & MUTED & EEF1E1 (+38084), PIP5K1P1 (+78409) \\
\hline 4 & cg02391410 & $1.26 \mathrm{E}-04$ & 1.00E-04 & 0.052 & chr16:59790082 & LOC644649 & NONE \\
\hline 5 & cg10960055 & 1.49E-03 & $1.00 \mathrm{E}-04$ & -0.083 & chr6:28664226 & & SCAND3 (-109115), TRIM27 (+227541) \\
\hline 6 & $\operatorname{cg} 17121197$ & $1.38 \mathrm{E}-03$ & 1.10E-03 & 0.06 & chr12:117353009 & FBXW8 & FBXW8 (+4249), TESC (+184241) \\
\hline 7 & cg18407858 & 1.75E-03 & $1.50 \mathrm{E}-03$ & 0.065 & chrX:47074283 & UBA1 & CDK16 (-8133), UBA1 (+24085) \\
\hline 8 & cg08470863 & $1.86 \mathrm{E}-03$ & 2.40E-03 & 0.064 & chr3:77524776 & $\mathrm{ROBO} 2$ & $\mathrm{ROBO} 2(+435483)$ \\
\hline 9 & cg24161397 & 1.89E-03 & 1.90E-03 & 0.064 & chr10:135038334 & KNDC1 & UTF1 (-5443), KNDC1 (+64364) \\
\hline 10 & cg07557707 & $1.16 \mathrm{E}-03$ & $1.20 \mathrm{E}-03$ & 0.054 & chr19:58549711 & ZSCAN1 & ZNF135 (-20895), ZSCAN1 (+4278) \\
\hline
\end{tabular}

Note: Ranked by a combination of both mean absolute difference in methylation level $(\beta)$ and statistical significance. DMPs = differentially methylated positions; MZ = monozygotic; hg19 = Human Genome version 19; GREAT = Genomic Regions Enrichment of Annotations Tool; TSS = transcription start site.

TABLE 2

Gene Ontology Enrichment Analysis for 500 Top-Ranked Diurnal Preference Associated DMPs

\begin{tabular}{|c|c|c|c|c|}
\hline GO accession ID & GO function & Ontology & $p$ value & $q$ value \\
\hline GO:0007156 & Homophilic cell adhesion via plasma membrane adhesion molecules & $\mathrm{BP}$ & 7.39E-13 & 2.91E-09 \\
\hline GO:0098742 & Cell-cell adhesion via plasma-membrane adhesion molecules & $\mathrm{BP}$ & 2.49E-11 & 3.37E-08 \\
\hline GO:0098609 & Cell-cell adhesion & $\mathrm{BP}$ & $2.56 \mathrm{E}-11$ & 3.37E-08 \\
\hline GO:0005509 & Calcium ion binding & MF & 4.70E-07 & 4.64E-04 \\
\hline GO:0007155 & Cell adhesion & $\mathrm{BP}$ & 1.04E-04 & 0.074 \\
\hline GO:0022610 & Biological adhesion & $\mathrm{BP}$ & 1.13E-04 & 0.074 \\
\hline GO:0090257 & Regulation of muscle system process & $\mathrm{BP}$ & $1.41 \mathrm{E}-03$ & 0.794 \\
\hline GO:0060004 & Reflex & $\mathrm{BP}$ & 4.11E-03 & 0.985 \\
\hline
\end{tabular}

Note: Ranked by $p$ value. $\mathrm{GO}=$ gene ontology, $\mathrm{DMPs}=$ differentially methylated positions; $\mathrm{BP}=$ biological process; MF = molecular function.

and downstream of LRPTM4 (gene encoding leucine rich repeat transmembrane neuronal 4 gene) with an established role in excitatory synapse development. The top 100 ranked diurnal preference associated DMPs are detailed in Table S2. Pyrosequencing confirmed a high correlation $(r=0.95)$ between DNA methylation levels detected using the Infinium microarray and pyrosequencing platforms (Figure 2; Figure S1). DMR analysis with comb-p found no consistent regions significantly differentially methylated between the twin-pairs. However, we identified three regions where more than one probe feature in the top 500 ranked probes and had a $\Delta \beta$ in the same direction. Probes $\operatorname{cg} 10960055(\Delta \beta-0.08, p=1.49 \mathrm{E}-03)$ and $\operatorname{cg} 10582608(\Delta \beta$ $-0.08, p=7.71 \mathrm{E}-03)$ are located in a cluster of tRNA genes located at 6p22.1, which was the region validated by Pyrosequencing. Two top ranked probes were located in the gene encoding for HDAC4, cg20149840 $(\Delta \beta 0.04, p=3.58 \mathrm{E}-$ $03)$, and $\operatorname{cg} 22077197(\Delta \beta 0.06, p=1.78 \mathrm{E}-02)$, and a further two probes, $\operatorname{cg} 21400851(\Delta \beta 0.05, p=3.71 \mathrm{E}-03)$ and $\operatorname{cg} 01678472(\Delta \beta$ 0.04, $p=1.39 \mathrm{E}-03)$, were identified in KCTD2.

\section{Diurnal Preference Associated DMPs are Signifi- cantly Enriched in Cell Adhesion and Calcium Binding Pathways}

In addition to probe-wise analysis, GO term enrichment analysis was performed on the 500 top-ranked diurnal preference associated DMPs (see Supplementary Table S3 for a list of loci included in the analysis). We identified six false discovery rate (FDR; $q$ value $<0.1$ ) and eight nominally significantly enriched terms $\left(p<5 \times 10^{-3}\right.$; see Table 2), including categories related to cell adhesion processes (GO:0007156, $p=7.39 \mathrm{E}-13$; GO:0098742, $p=2.49 \mathrm{E}$ 11; GO:0098609, $p=2.56 \mathrm{E}-11$; GO:0007155, $p=1.04 \mathrm{E}-04$ ) and calcium ion binding (GO:0005509, $p=4.70 \mathrm{E}-07$ ).

\section{Discussion}

The present study is the first comprehensive genome-wide analysis of DNA methylation differences related to diurnal preference using a discordant MZ twin design. We identified a substantial number of probes that were differentially methylated in twins with high eveningness as compared to their co-twins with low eveningness. A propensity for eveningness has been associated with a range of behavioral traits, including lower psychological well-being (Wittmann et al., 2010) and an increase in substance use such as tobacco, alcohol, and illicit drugs (Urban et al., 2011; Wittmann et al., 2010).

One of the highest ranked DMPs (cg23881566) is hypomethylated ( $\Delta \beta-0.06, p=7.29 \mathrm{E}-04)$ in the twins with substantially higher eveningness scores than their co-twin. This CpG is located in the first intron of the gene encoding SRRM4 situated within a DNAse I-hypersensitive region, suggesting that the chromatin in this region is open and thus possibly regulatory or transcriptionally active. SRRM 4 
cg07409153

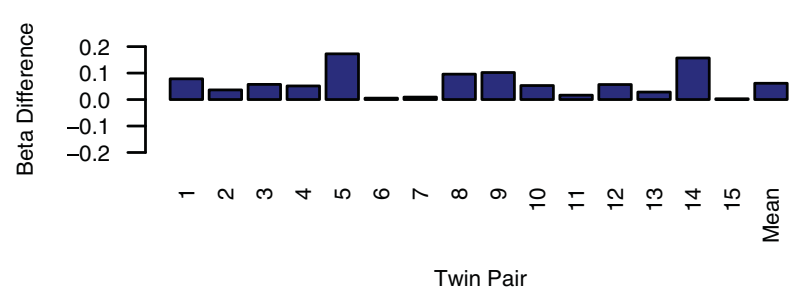

cg06142043

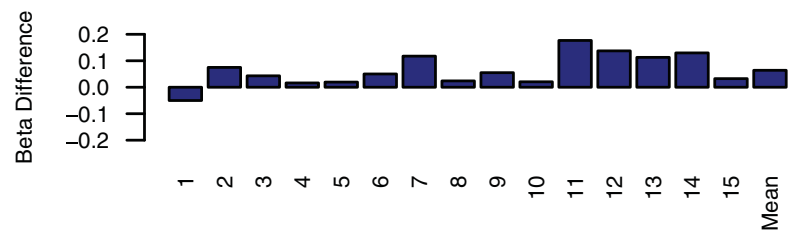

Twin Pair

cg10960055

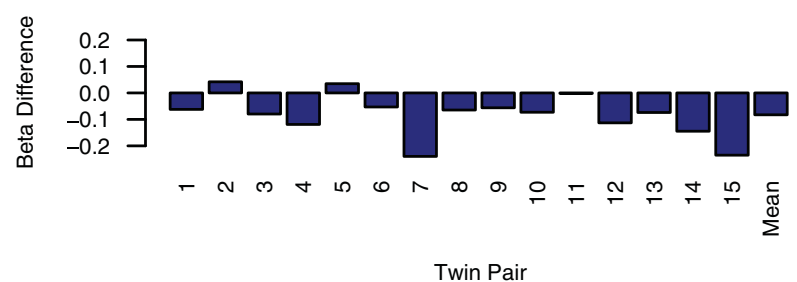

cg18407858

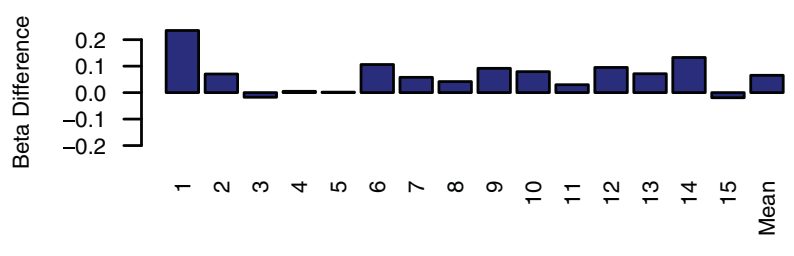

Twin Pair

cg24161397

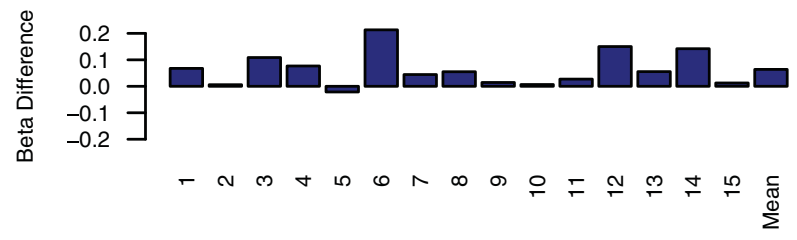

Twin Pair cg23881566

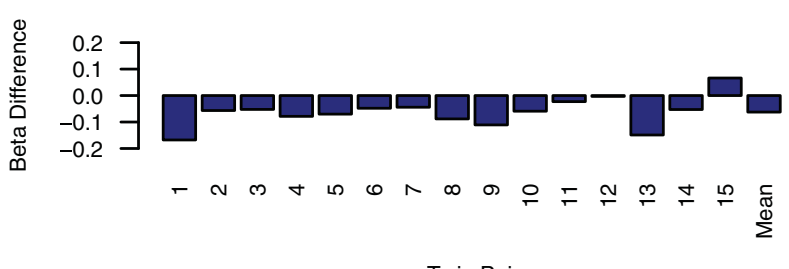

cg02391410

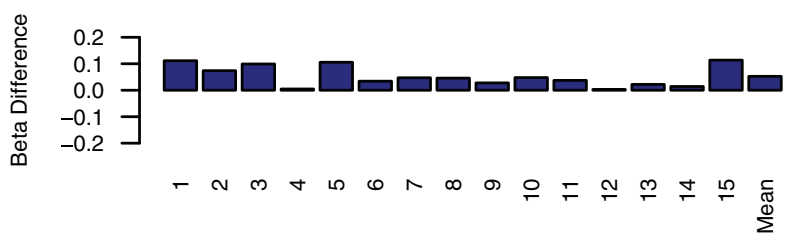

Twin Pair

cg17121197

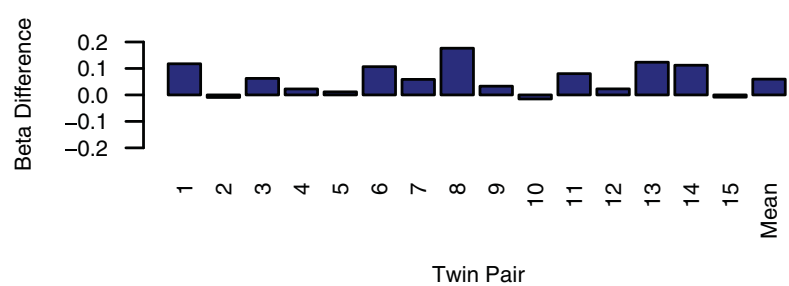

cg08470863

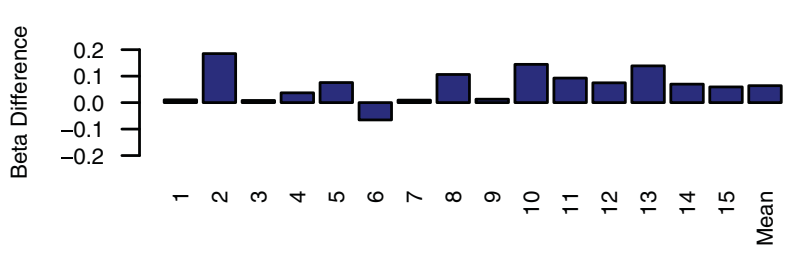

Twin Pair

cg07557707

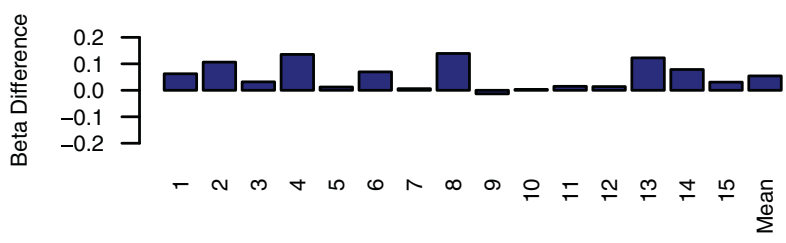

Twin Pair

\section{Figure 1}

(Colour online) Difference in DNA methylation ( $\beta$ value) between twins for the ten top-ranked probes (twins with eveningness preference - cotwin with morningness preference).

is highly expressed in the brain and its misregulation has been implicated in the autistic brain (Irimia et al., 2014); interestingly, circadian rhythm irregularities are often observed in autism spectrum disorders (Tordjman et al., 2015). The 9th-ranked probe is located in the last coding exon of KNDC1. Endothelial cells engineered to express reduced
KNDC1 (knockdown) show delayed senescence (Zhang et al., 2014); cellular senescence impairs circadian rhythmicity both in vitro and in vivo (Kunieda et al., 2006). Thus, both SRRM4 and KNDC1 may warrant further investigation in relation to epigenetic regulation of diurnal preference. 


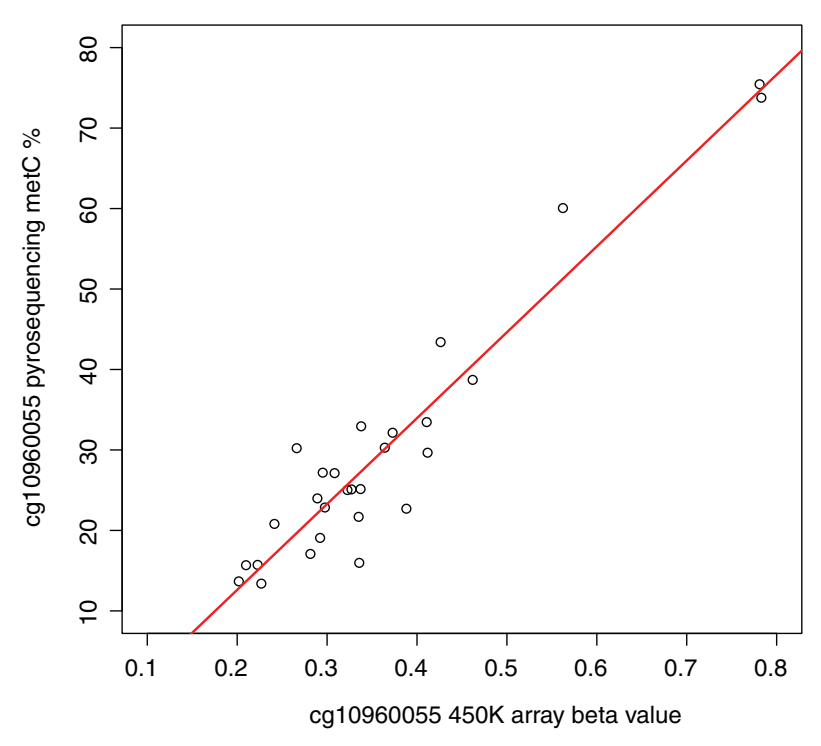

Figure 2

(Colour online) Relationship between beta values from the $450 \mathrm{~K}$ array and bisulfite pyrosequencing for one of the top-ranked differentially methylated probes cg10960055 $(r=0.95)$.

Downstream GO enrichment analysis on the 500 topranked diurnal preference associated DMPs identified a number of FDR significant over-represented terms, including cell adhesion processes and calcium ion binding. These two categories are of particular interest as cell adhesion molecules are integral to the maintenance of the circadian circuitry (Kon et al., 2014; Nitabach et al., 2005) and dynamic fluctuation of ions, especially $\mathrm{Ca}^{2+}$, appears to be essential for circadian clockwork (Brancaccio et al., 2013; Nitabach et al., 2005). Further, genetic studies in sleep and the circadian system have associated calcium signaling as one of the key biological pathways involved (Parsons, 2015).

Despite the power of the discordant MZ twin approach for epigenetic epidemiology, there are several limitations to this study. Due to the relatively small sample size and effect sizes detected, our single-probe analysis did not report any diurnal-preference associated DMP that reached Bonferroni-corrected levels of significance ( $p<1.10 \mathrm{E}-07)$. However, this statistical approach is likely to be too conservative, especially given the known non-independence of DNA methylation across the probes represented on the array (Rakyan et al., 2011b). In fact, DNA methylation studies in other psychiatric phenotypes (and complex disorders in general) report similarly small absolute differences compared to this study (Dempster et al., 2011; Dempster et al., 2014; Rakyan et al., 2011a; Wong et al., 2014) and the combined analytical approach used (which took into account the significance and the extent of methylation change) has been reported to produce gene lists of higher reproducibility and biological relevance compared with the conventional method that relies solely on statistical significance in previ- ous DNA methylation and gene expression studies (Dempster et al., 2011; Dempster et al., 2014; Kadota et al., 2009; Wong et al., 2014). One further caveat is that the MEQ was completed by the participants on average 18 months after the DNA samples were collected; however, recent analysis on the diurnal preference measures at different waves in this cohort have found the measure to be relatively stable across time-points separated by five years ( $r=0.64$; Barclay et al., 2015). Nonetheless, given the relatively small sample size, replication in larger samples is needed.

In conclusion, we have identified DNA methylation differences associated with diurnal preference in peripheral DNA samples from discordant MZ twin-pairs. The data generated in this study need to be replicated but are suggestive of a role for epigenetic variation in diurnal preference that is independent of the underlying DNA sequence.

\section{Acknowledgments}

This work was supported by a grant awarded by the Psychiatric Research Trust (to TCE, JM, and ELD). The G1219 Study collection wave 4 was supported by the Economic and Social Research Council (Grant No. RES-000-22-2206) and the Institute of Social Psychiatry (Grant No. 06/07-11) to AMG. Samples' DNA extraction was supported by a Goldsmiths Early Career Award to AMG. This study presents independent research part funded by the National Institute for Health Research (NIHR) Biomedical Research Centre at South London and Maudsley NHS Foundation Trust and King's College London. The views expressed are those of the author(s) and not necessarily those of the NHS, the NIHR, or the Department of Health. We thank the families for their participation as well as numerous staff members and students from the Social Genetic Developmental Psychiatry Centre, Institute of Psychiatry, London, and Goldsmiths, University of London. The authors report no biomedical financial interests or potential conflicts of interest.

\section{Supplementary Material}

To view supplementary material for this article, please visit http://dx.doi.org/10.1017/thg.2015.78.

\section{References}

Archer, S. N., Carpen, J. D., Gibson, M., Lim, G. H., Johnston, J. D., Skene, D. J., \& von Schantz, M. (2010). Polymorphism in the PER3 promoter associates with diurnal preference and delayed sleep phase disorder. Sleep, 33, 695-701.

Azzi, A., Dallmann, R., Casserly, A., Rehrauer, H., Patrignani, A., Maier, B., ... Brown, S. A. (2014). Circadian behavior is light-reprogrammed by plastic DNA methylation. Nature Neuroscience, 17, 377-382.

Barclay, N. L., Eley, T. C., Buysse, D. J., Archer, S. N., \& Gregory, A. M. (2010). Diurnal preference and sleep quality: Same genes? A study of young adult twins. Chronobiology International, 27, 278-296. 
Barclay, N. L., Rowe, R., O'Leary, R., Bream, D., \& Gregory, A. M. (2015). Stability of genetic and environmental influences on the association between diurnal preference and sleep quality over time in young adult twins and siblings. Manuscript submitted for publication.

Belden, W. J., Lewis, Z. A., Selker, E. U., Loros, J. J., \& Dunlap, J. C. (2011). CHD1 remodels chromatin and influences transient DNA methylation at the clock gene frequency. PLoS Genetics, 7, e1002166.

Bell, J. T., \& Spector, T. D. (2011). A twin approach to unraveling epigenetics. Trends in Genetics, 27, 116-125.

Brancaccio, M., Maywood, E. S., Chesham, J. E., Loudon, A. S., \& Hastings, M. H. (2013). A Gq-Ca2+ axis controls circuitlevel encoding of circadian time in the suprachiasmatic nucleus. Neuron, 78, 714-728.

Chen, Y. A., Choufani, S., Grafodatskaya, D., Butcher, D. T., Ferreira, J. C., \& Weksberg, R. (2012). Cross-reactive DNA microarray probes lead to false discovery of autosomal sexassociated DNA methylation. American Journal of Human Genetics, 91, 762-764.

Davis, S., Du, P., Bilke, S., Triche, T. Jr., \& Bootwalla, M. (2015). methylumi: Handle Illumina methylation data. R package version 2.16.0.

Dempster, E. L., Pidsley, R., Schalkwyk, L. C., Owens, S., Georgiades, A., Kane, F., ... Mill, J. (2011). Diseaseassociated epigenetic changes in monozygotic twins discordant for schizophrenia and bipolar disorder. Human Molecular Genetics, 20, 4786-4796.

Dempster, E. L., Wong, C. C., Lester, K. J., Burrage, J., Gregory, A. M., Mill, J., \& Eley, T. C. (2014). Genomewide methylomic analysis of monozygotic twins discordant for adolescent depression. Biological Psychiatry, 76, 977983.

Dijk, D. J., \& Lockley, S. W. (2002). Integration of human sleep-wake regulation and circadian rhythmicity. Journal of Applied Physiology, 92, 852-862.

Duffy, J. F., Rimmer, D. W., \& Czeisler, C. A. (2001). Association of intrinsic circadian period with morningnesseveningness, usual wake time, and circadian phase. Behavioral Neuroscience, 115, 895-899.

Feng, D., Liu, T., Sun, Z., Bugge, A., Mullican, S. E., Alenghat, T., ... Lazar, M. A. (2011). A circadian rhythm orchestrated by histone deacetylase 3 controls hepatic lipid metabolism. Science, 331, 1315-1319.

Freeman, B., Smith, N., Curtis, C., Huckett, L., Mill, J., \& Craig, I. W. (2003). DNA from buccal swabs recruited by mail: Evaluation of storage effects on long-term stability and suitability for multiplex polymerase chain reaction genotyping. Behavior Genetics, 33, 67-72.

Gau, S. S., Shang, C. Y., Merikangas, K. R., Chiu, Y. N., Soong, W. T., \& Cheng, A. T. (2007). Association between morningness-eveningness and behavioral/emotional problems among adolescents. Journal of Biological Rhythms, 22, 268-274.

Gau, S. S., Soong, W. T., \& Merikangas, K. R. (2004). Correlates of sleep-wake patterns among children and young adolescents in Taiwan. Sleep, 27, 512-519.
Gregory, A. M., Buysse, D. J., Willis, T. A., Rijsdijk, F. V., Maughan, B., Rowe, R., ... Eley, T. C. (2011). Associations between sleep quality and anxiety and depression symptoms in a sample of young adult twins and siblings. Journal of Psychosomatic Research, 71, 250-255.

Heijmans, B. T., \& Mill, J. (2012). Commentary: The seven plagues of epigenetic epidemiology. International Journal of Epidemiology, 41, 74-78.

Horne, J. A., \& Ostberg, O. (1976). A self-assessment questionnaire to determine morningness-eveningness in human circadian rhythms. International Journal of Chronobiology, 4, 97-110.

Irimia, M., Weatheritt, R. J., Ellis, J. D., Parikshak, N. N., Gonatopoulos-Pournatzis, T., Babor, M., ... Blencowe, B. J. (2014). A highly conserved program of neuronal microexons is misregulated in autistic brains. Cell, 159, 15111523.

Kadota, K., Nakai, Y., \& Shimizu, K. (2009). Ranking differentially expressed genes from Affymetrix gene expression data: Methods with reproducibility, sensitivity, and specificity. Algorithms for Molecular Biology, 4, 7.

Katzenberg, D., Young, T., Finn, L., Lin, L., King, D. P., Takahashi, J. S., \& Mignot, E. (1998). A CLOCK polymorphism associated with human diurnal preference. Sleep, 21, 569-576.

Kerkhof, G. A. (1985). Inter-individual differences in the human circadian system: A review. Biological Psychology, 20, 83-112.

Kon, N., Yoshikawa, T., Honma, S., Yamagata, Y., Yoshitane, H., Shimizu, K., ... Fukada, Y. (2014). CaMKII is essential for the cellular clock and coupling between morning and evening behavioral rhythms. Genes \& Development, 28, 1101-1110.

Koskenvuo, M., Hublin, C., Partinen, M., Heikkila, K., \& Kaprio, J. (2007). Heritability of diurnal type: A nationwide study of 8753 adult twin pairs. Journal of Sleep Research, 16, 156-162.

Kunieda, T., Minamino, T., Katsuno, T., Tateno, K., Nishi, J., Miyauchi, H., ... Komuro, I. (2006). Cellular senescence impairs circadian expression of clock genes in vitro and in vivo. Circulation Research, 98, 532-539.

McAdams, T. A., Gregory, A. M., \& Eley, T. C. (2013). Genes of experience: Explaining the heritability of putative environmental variables through their association with behavioural and emotional traits. Behavior Genetics, 43, 314-328.

Mongrain, V., Lavoie, S., Selmaoui, B., Paquet, J., \& Dumont, M. (2004). Phase relationships between sleep-wake cycle and underlying circadian rhythms in morningnesseveningness. Journal of Biological Rhythms, 19, 248-257.

Nitabach, M. N., Sheeba, V., Vera, D. A., Blau, J., \& Holmes, T. C. (2005). Membrane electrical excitability is necessary for the free-running larval Drosophila circadian clock. Journal of Neurobiology, 62, 1-13.

Palagini, L., Biber, K., \& Riemann, D. (2014). The genetics of insomnia-Evidence for epigenetic mechanisms? Sleep Medicine Reviews, 18, 225-235. 
Parsons, M. (2015). On the genetics of sleep disorders: GWAS and beyond. Advances in Genomics and Genetics, 20, 293303.

Pedersen, B. S., Schwartz, D. A., Yang, I. V., \& Kechris, K. J. (2012). Comb-p: Software for combining, analyzing, grouping and correcting spatially correlated P-values. Bioinformatics, 28, 2986-2988.

Pidsley, R., Wong, C. C. Y., Volta, M., Lunnon, K., Mill, J., \& Schalkwyk, L. C. (2013). A data-driven approach to preprocessing Illumina $450 \mathrm{~K}$ methylation array data. BMC Genomics, 14, 293.

Price, M. E., Cotton, A. M., Lam, L. L., Farre, P., Emberly, E., Brown, C. J., ... Kobor, M. S. (2013). Additional annotation enhances potential for biologically-relevant analysis of the Illumina Infinium HumanMethylation450 BeadChip array. Epigenetics Chromatin, 6, 4.

Qureshi, I. A., \& Mehler, M. F. (2014). Epigenetics of sleep and chronobiology. Current Neurology and Neuroscience Reports, 14, 432.

Rakyan, V. K., Beyan, H., Down, T. A., Hawa, M. I., Maslau, S., Aden, D., ... Leslie, R. D. (2011a). Identification of type 1 diabetes-associated DNA methylation variable positions that precede disease diagnosis. PLoS Genet, 7, e1002300.

Rakyan, V. K., Down, T. A., Balding, D. J., \& Beck, S. (2011b). Epigenome-wide association studies for common human diseases. Nature Reviews Genetics, 12, 529-541.

Randler, C. (2011). Association between morningnesseveningness and mental and physical health in adolescents. Psychology, Health \& Medicine, 16, 29-38.

Sandoval, J., Heyn, H., Moran, S., Serra-Musach, J., Pujana, M. A., Bibikova, M., \& Esteller, M. (2011). Validation of a DNA methylation microarray for $450000 \mathrm{CpG}$ sites in the human genome. Epigenetics, 6, 692-702.

Toh, K. L., Jones, C. R., He, Y., Eide, E. J., Hinz, W. A., Virshup, D. M., .. Fu, Y. H. (2001). An hPer2 phosphorylation site mutation in familial advanced sleep phase syndrome. Science, 291, 1040-1043.

Tordjman, S., Davlantis, K. S., Georgieff, N., Geoffray, M. M., Speranza, M., Anderson, G. M., ... Dawson, G. (2015). Autism as a disorder of biological and behavioral rhythms: Toward new therapeutic perspectives. Frontiers in Pediatrics, 3,1 .

Urban, R., Magyarodi, T., \& Rigo, A. (2011). Morningnesseveningness, chronotypes and health-impairing behaviors in adolescents. Chronobiology International, 28, 238-247.

von Schantz, M., Taporoski, T. P., Horimoto, A. R., Duarte, N. E., Vallada, H., Krieger, J. E., ... Pereira, A. C. (2015). Distribution and heritability of diurnal preference (chronotype) in a rural Brazilian family-based cohort, the Baependi study. Scientific Reports, 5, 9214.

Wittmann, M., Paulus, M., \& Roenneberg, T. (2010). Decreased psychological well-being in late 'chronotypes' is mediated by smoking and alcohol consumption. Subst Use Misuse, 45, 15-30.

Wong, C. C., Meaburn, E. L., Ronald, A., Price, T. S., Jeffries, A. R., Schalkwyk, L. C., ... Mill, J. (2014). Methylomic analysis of monozygotic twins discordant for autism spectrum disorder and related behavioural traits. Molecular Psychiatry, 19, 495-503.

Young, M. D., Wakefield, M. J., Smyth, G. K., \& Oshlack, A. (2010). Gene ontology analysis for RNA-seq: Accounting for selection bias. Genome Biology, 11, R14.

Zhang, C., Zhen, Y. Z., Lin, Y. J., Liu, J., Wei, J., Xu, R., \& Hu, G. (2014). KNDC1 knockdown protects human umbilical vein endothelial cells from senescence. Molecular Medicine Reports, 10, 82-88.

Zhang, E. E., \& Kay, S. A. (2010). Clocks not winding down: Unravelling circadian networks. Nature Reviews Molecular Cell Biology, 11, 764-776. 